\title{
Feasibility of microscope-integrated swept-source optical coherence tomography in canaloplasty
}

\author{
Huan Xu ${ }^{1,2 \#}$, Wangyi Fang ${ }^{1,2 \#}$, Guangxing Liu ${ }^{3}$, Jinyu Fan ${ }^{3}$, Jian Yu ${ }^{1,2}$, Yuan Zong ${ }^{1,2}$, Chunhui Jiang ${ }^{1,2}$, \\ Guohua $\mathrm{Shi}^{3,4}$, Xinghuai Sun ${ }^{1,2}$ \\ ${ }^{1}$ Department of Ophthalmology and Visual Science, Eye, Ear, Nose and Throat Hospital, Shanghai Medical College of Fudan University, Shanghai, \\ China; ${ }^{2}$ Key NHC Key Laboratory of Myopia (Fudan University), Laboratory of Myopia, Chinese Academy of Medical Sciences, Shanghai, China; \\ ${ }^{3}$ Jiangsu Key Laboratory of Medical Optics, Suzhou Institute of Biomedical Engineering and Technology, Chinese Academy of Sciences, Suzhou, \\ China; ${ }^{4}$ CAS Center for Excellence in Brain Science and Intelligence Technology, Shanghai, China \\ "These authors contributed equally to this work as co-first authors. \\ Correspondence to: Chunhui Jiang. Department of Ophthalmology and Vision Science, Eye and ENT Hospital, Fudan University, 83 Fenyang Rd, \\ Shanghai 200031, China. Email: chhjiang70@163.com; Guohua Shi. Jiangsu Key Laboratory of Medical Optics, Suzhou Institute of Biomedical \\ Engineering and Technology, Chinese Academy of Sciences, 88 Kelin Rd, Suzhou 215163, China. Email: ghshi_lab@126.com.
}

Background: Several researchers have used commercial microscope-integrated optical coherence tomography (OCT) systems in glaucoma surgery, including ab interno trabeculectomy and canaloplasty. However, the $840 \mathrm{~nm}$ wavelength light source of the OCT systems is not ideal for imaging the anterior chamber angle structures because of its limited penetration. We evaluated the potential value of a microscope-integrated swept-source OCT system with a $1,310 \mathrm{~nm}$ center-wavelength light in canaloplasty for glaucoma.

Methods: Sixteen porcine eyes were used to simulate canaloplasty. The critical surgical steps were monitored using a prototype microscope-integrated OCT system with a 1,310 nm light source and a high axial scan rate of $100 \mathrm{kHz}$. The images from swept-source OCT and three-dimensional images from the microscope were projected simultaneously onto a liquid crystal display three-dimensional monitor (LMD4251TD, Sony, Japan). The changes in the collector vessel (aqueous drainage structure in the porcine eye, similar to Schlemm's canal in humans) were measured using Image J software. Histological sections stained with hematoxylin and eosin were used to assess surgical efficacy.

Results: High-resolution real-time images of the anterior segment were acquired during canaloplasty using the microscope-integrated OCT system. With the real-time OCT images, the position of the collector vessel was identified and the scleral flap could be created at the ideal location. The expansion of the collector vessel after viscoelastic injection was also visualized in real time. Compared with baseline, there was a significant increase in the cross-sectional area (from $14,502.98 \pm 9,242.55$ to $59,499.04 \pm 20,506.41 \mu \mathrm{m}^{2}, \mathrm{P}<0.001$ ) of the collector vessel.

Conclusions: Using the microscope-integrated OCT system, real-time images of the anterior segment were successfully acquired during the operation. The microscope-integrated OCT system might be useful in future anti-glaucoma surgery.

Keywords: Anterior segment; MIOCT; canaloplasty; intraoperative real-time imaging

Submitted Apr 22, 2020. Accepted for publication Oct 10, 2020.

doi: 10.21037/atm-20-3469

View this article at: http://dx.doi.org/10.21037/atm-20-3469 


\section{Introduction}

Glaucoma is a leading cause of irreversible blindness worldwide (1). Despite the development of antiglaucoma medications, surgery remains the mainstay of treatment (2). Microinvasive antiglaucoma surgery has recently gained favor among ophthalmologists, and viscoelastic canaloplasty is the preferred technique. Introduced recently, viscoelastic canaloplasty is a modern form of non-penetrating glaucoma surgery (3-5). Multiple follow-up studies have revealed that this surgery may achieve sustained, long-term reductions in intraocular pressure (IOP) in eyes with primary open-angle glaucoma (4,6-10).

Compared with traditional methods, canaloplasty lowers IOP with minimal complications or iatrogenic damage $(1,11)$. However, this procedure is technically demanding, with a long learning curve, especially in terms of locating Schlemm's canal (12). On the other hand, optical coherence tomography (OCT) can provide high-resolution crosssectional images of the eye without requiring contact. In 2009, Dayani et al. reported the application of handheld OCT in ocular surgery for the first time (13). Since then, OCT has been used to guide various forms of ocular surgery (14-17). With further optimization of the technique, a breakthrough was achieved in the form of real-time imaging during vitreoretinal surgery through microscope-integrated OCT (MIOCT), first reported by Binder et al. (18). Subsequently, several researchers have used commercial MIOCT systems (iOCT, OptoMedical Technologies, Luebeck, Germany; Rescan 700, Carl Zeiss Meditec, Jena, Germany) in glaucoma surgery, including ab interno trabeculectomy and canaloplasty (19-21). The systems include a built-in heads-up display, which provides realtime intraoperative OCT feedback without interrupting the surgery. However, the $840 \mathrm{~nm}$ wavelength light source of the OCT systems is not ideal for imaging the structures of the anterior chamber angle because its penetration is relatively limited.

In the present study, we used a prototype MIOCT system with a $1,310 \mathrm{~nm}$ center-wavelength swept-source light, which achieves deep penetration and real-time imaging with a high signal-to-noise ratio (22). Using this system, a three-dimensional (3D) surgical image from the microscope and the OCT image of the tissue level could be projected together onto the same screen. Here, we simulated surgery in porcine eyes to investigate the potential value of our MIOCT system in canaloplasty.

We present the following article in accordance with the
ARRIVE reporting checklist (available at http://dx.doi. org/10.21037/atm-20-3469).

\section{Methods}

The study was approved by the Institutional Review Board of the Eye and ENT Hospital of Fudan University, Shanghai, China (No. 2019031-1). Eight male Yorkshire pigs (10 kg, 2 months old, clean grade) were purchased from Jiagan Biotechnology Co., Ltd. and maintained in a $12 \mathrm{~h} \mathrm{light/dark} \mathrm{cycle} \mathrm{with} \mathrm{free} \mathrm{access} \mathrm{to} \mathrm{food} \mathrm{and} \mathrm{water.}$ After the Yorkshire pigs were acclimatized for at least 7 days, they were used in accordance with the Association for Research in Vision and Ophthalmology Statement for the Use of Animals in Ophthalmic and Vision Research. Canaloplasty was performed on the sixteen eyes of eight Yorkshire pigs. The parameters before and after the operation were acquired and analyzed as self-control.

\section{MIOCT system}

In the MIOCT prototype, swept-source OCT (SS-OCT) was optically designed for use in conjunction with the optical path of a microscope. The microscope and OCT shared the same objective lens, with a focal length of $200 \mathrm{~mm}$. SSOCT used the $1,310 \mathrm{~nm}$ wavelength range at a high axial scan rate of $100 \mathrm{kHz}$ and a high-speed tunable laser (HSL20, Santec, Japan). Its lateral resolution was $14 \mu \mathrm{m}$, and its lateral range was $10.7 \mathrm{~mm}$ with $768 \mathrm{~A}$-Scans/B-Scan. The axial resolution was $11 \mu \mathrm{m}$, and the maximum depth in air was $6 \mathrm{~mm}$. After detection, the OCT signals were processed with a computer (CPU: E5-2620v4@2.1 GHz; RAM: 32 GB; graphics cards: GTX1080Ti) to output real-time OCT images. A superluminescent diode with a wavelength of $640 \mathrm{~nm}$ was combined in the sample OCT arm and used to mark the OCT imaging regions. The binocular microscopic images were separated using a beam splitter. They were recorded with two surgical video cameras (MCC500MDC, Sony, Japan) and displayed on a high-definition (1080P; 1,920×1,080) 42-inch LCD 3D monitor (LMD4251TD, Sony). Real-time OCT images of the same region of interest are displayed in the lower right corner of the same screen. The surgeon wore circular polarizing 3D glasses to allow precise depth perception (Figure S1). The final time delays in the acquisition of the OCT image and dualchannel microscopic image were less than 120 and $100 \mathrm{ms,}$ respectively. 


\section{Surgical protocol}

Surgery was conducted on 16 porcine eyes from eight live male Yorkshire pigs (10 kg, 2 months old). After general anesthesia, the eyelids were opened. The real-time OCT images of (Figure 1A,B) the porcine drainage system was acquired. The porcine drainage system at the anterior chamber angle has a series of collector vessels or the angular aqueous plexus, which is similar to the Schlemm's canal in primates. Then, the conjunctiva and Tennon's membrane were removed. After the collector vessel was located with the MIOCT system, a $5 \times 5 \mathrm{~mm}^{2}$ limbal-based one-half thickness scleral flap was created. The scalpel was stopped when the flap exceeded the position of the collector vessel (Figure $1 C, D$ ). After that, an incision perpendicular to the limbus was made across the scleral bed and the collector vessel; care was taken not to penetrate the inner wall of the collector vessel. The opening of the collector vessel was then expanded with a custom-made trabeculotome (Figure S2) with the tip adapted from an optical fiber $(\Phi=250 \mu \mathrm{m})$ (Figure 1E,F). An optical fiber microcatheter $(\Phi=200 \mu \mathrm{m})$ (iTrack 250, Ellex iScience, Inc., Freemont, CA, USA) connected to a red flickering laser light was then used to identify the location of the fiber tip, which had been threaded into the collector vessel. When the distal end of the microcatheter reached the terminal site, the viscoelastic (DisCoVisc, Alcon Laboratories, Fort Worth, USA) was injected via a screw-driven syringe connected to the microcatheter. This screw-driven syringe was twisted again as the microcatheter was withdrawn in a circle with an angle of 30 degrees (Figure 1G,H). According to the $3 \mathrm{Rs}$ principle, all the animals were euthanized at the end of the experiment by intracardial injection of approximately $20 \mathrm{~mL}$ of $15 \%$ potassium chloride solution. Their eyes were then rapidly enucleated for histopathological evaluation.

\section{MIOCT imaging and measurement}

Intraoperative MIOCT images were acquired at the beginning of surgery and after viscoelastic injection for further analysis. The scanning area could be controlled by the surgeon by moving the microscope. In the crosssectional OCT images, the collector vessel appeared as a dark slit and oval structure. After the surgery, the collector vessels in each of the acquired image were outlined manually by two surgeons ( $\mathrm{XH}$ and $\mathrm{FW}$ ) and their areas were automatically quantified using Image J software (http:// imagej.nih.gov/ij/; National Institutes of Health, Bethesda,
MD, USA). Two surgeons, who were blinded to all sample identities, performed the measurements separately. The average values were used for subsequent analyses.

\section{Statistical analyses}

All statistical analyses were conducted using SPSS version 20.0 (SPSS, Inc., Chicago, IL, USA). All variables were checked for conformation to a normal distribution with the one-sample Kolmogorov-Smirnov test. The cross-sectional area of the collector vessel before and after surgery was compared using paired-sample $t$-tests.

\section{Results}

Surgery was conducted on 16 porcine eyes from eight healthy Yorkshire pigs. High-resolution real-time images of the anterior segment of the eyes were acquired intraoperatively with the MIOCT system. The collector vessels were located according to the OCT images. The collector vessel lumina had a low signal, whereas the walls had a relatively highly reflective signal.

With the guidance of the images, the scleral flap was created until its tip exceeded the collector vessel. With the collector vessels visible, the incision was made across the scleral bed. In one eye, the scalpel unexpectedly penetrated the anterior chamber while making the incision and this eye was excluded. A flexible microcatheter was then threaded into the collector vessels. After viscoelastic injection, the collector vessel lumen was enlarged in OCT images of all 15 porcine eyes (Figures $1 H, 2$ ). The results demonstrated that there was significant increase, compared with baseline, in the area (from $14,502.98 \pm 9,242.55$ to $\left.59,499.04 \pm 20,506.41 \mu^{2}, \mathrm{P}<0.001\right)$ of the collector vessels (Figure 3). For the measurement of collector vessel area, the intraclass correlation coefficient (ICC) values indicated good consistency between the two readers (Table S1). Histopathological assessment confirmed the expansion of the collector vessels (Figure 4).

\section{Discussion}

This study demonstrates the feasibility of using MIOCT with a 1,310 nm wavelength swept-source light source in canaloplasty. Real-time high-resolution images of the anterior segmental structures were acquired during the procedure. These images provided real-time information for surgeons to make decisions during the procedure and 

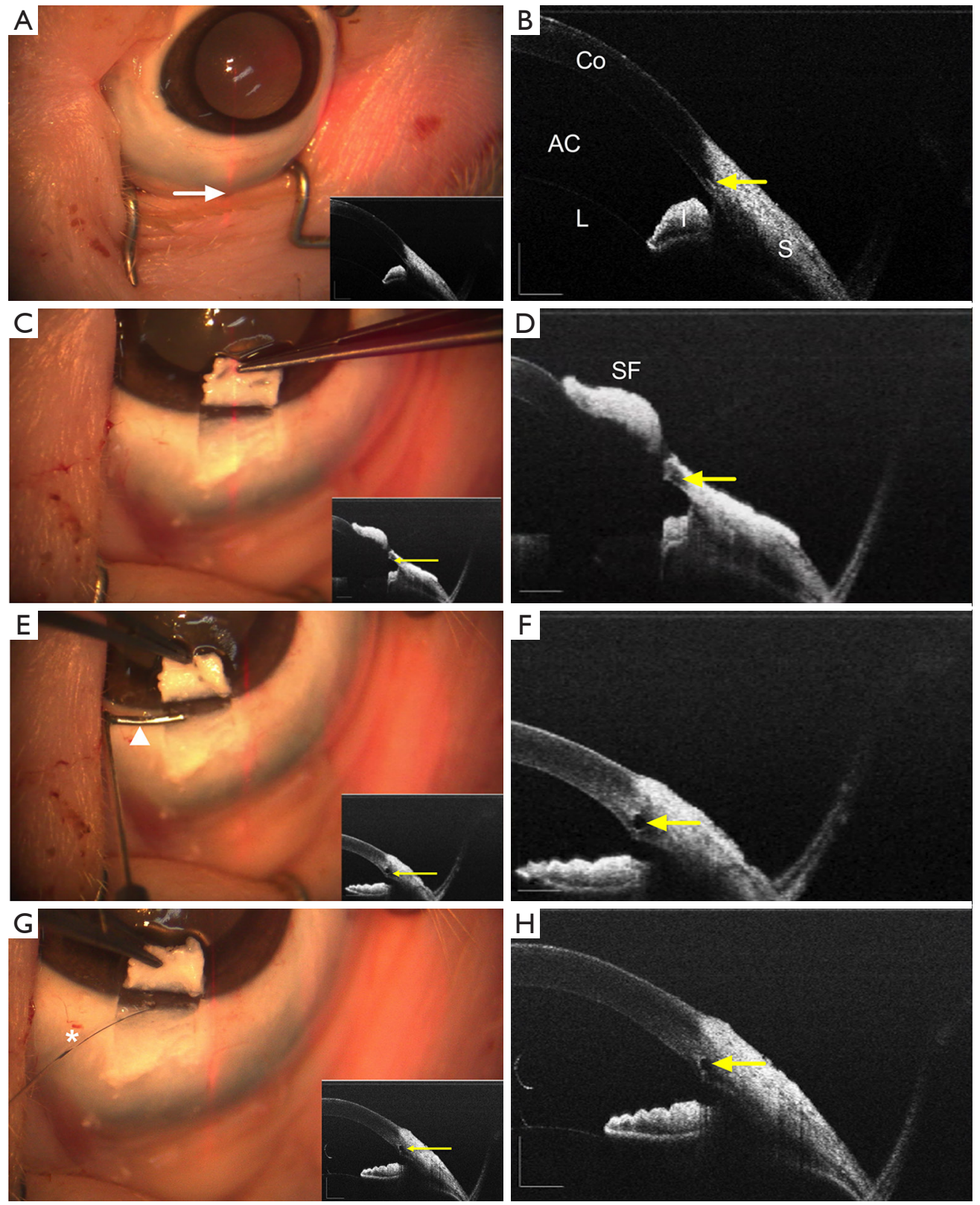

Figure 1 Surgical procedure of canaloplasty and its corresponding real-time images from the microscope-integrated swept-source optical coherence tomography (MIOCT) system. (A) MIOCT image before surgery; (B) cross-sectional OCT image of the red scanning line (white arrow) clearly shows the anterior segmental structures (AC: anterior chamber; Co: cornea; L: lens; I: iris; S: sclera) and collector vessel (yellow arrow); (C) MIOCT image after incision of a superficial scleral flap; (D) OCT image shows the location of the collector vessel and scleral flap (SF); (E) MIOCT image after insertion of a custom-made trabeculotome with an optical fiber tip (white arrowhead); (F) expansion of the collector vessel was observed on the OCT image; (G) MIOCT image after insertion of a flexible microcatheter (white asterisk) and viscoelastic was injected; (H) OCT image confirming expansion of the collector vessel. 

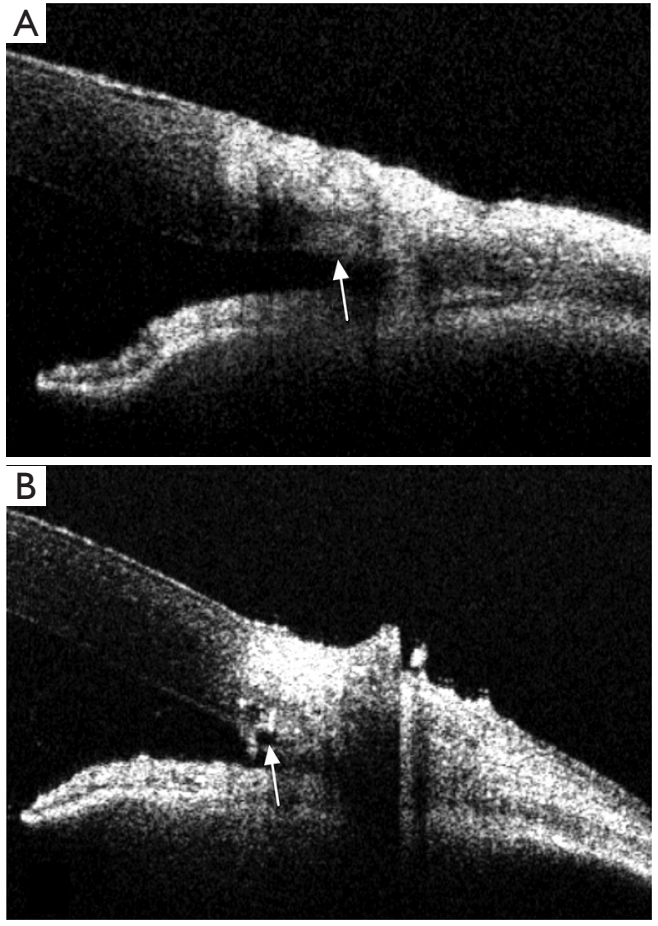

Figure 2 Changes in the lumen of the collector vessel before (A) and after (B) viscoelastic injection during canaloplasty. White arrowheads show the location of collector vessel.

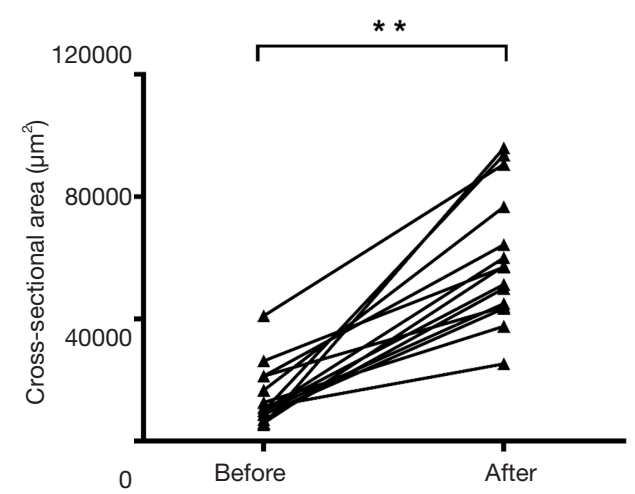

Figure 3 Quantitative evaluation of the cross-sectional area before and after canaloplasty. ${ }^{* *}, \mathrm{P}<0.01$, tested with paired-sample $t$-tests.

also confirmed the expansion of the collector vessel.

Precise and minimally invasive approaches have become popular in modern surgery, especially in ophthalmology. High-resolution, intraoperative real-time imaging is one way to ensure that these surgeries are successful $(2,23)$. In recent years, roentgenograms and B-mode ultrasound

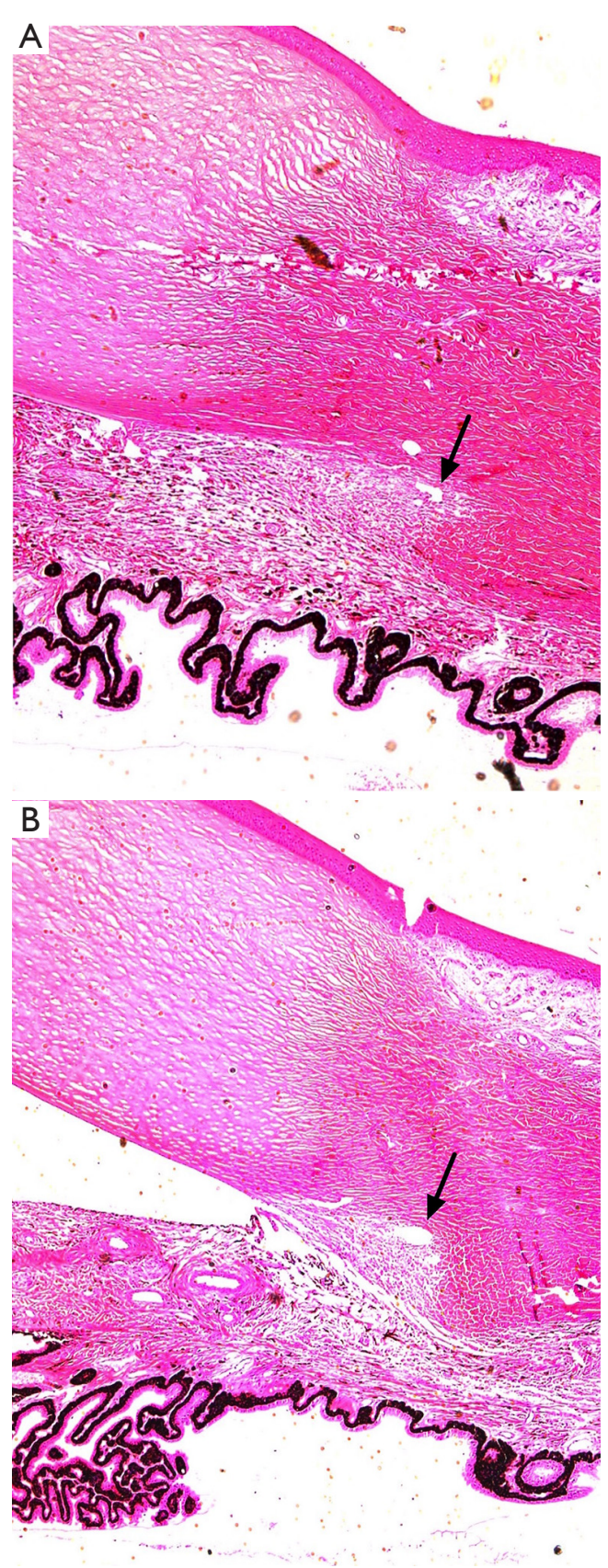

Figure 4 Hematoxylin and eosin staining of histological sections obtained before (A) and after (B) canaloplasty (20x).

have been used and yielded precise and predictable results in prior studies (24-27). In ophthalmology, OCT has been used for several years, and has greatly increased our understanding of many ocular structures. Sweptsource OCT was recently introduced and is capable of acquiring a B-scan in $10 \mathrm{~ms}$, which allows intraoperative 
real-time imaging. With the guidance of MIOCT system intraoperatively, the present study showed that highresolution real-time images of the anterior segment of the eye during canaloplasty could be acquired.

Previous studies have reported that the success rate of cannulation was about $74-89.9 \%(3,4,28,29)$. Furthermore, in glaucomatous eyes, anatomical anomalies in Schlemm's canal, arising from pathological changes or previous surgeries, can make cannulation even more challenging $(6,8,10)$, especially for beginners. In our study, to test the feasibility and potential value of the MIOCT system in guiding canaloplasty, all steps in the surgical procedure were simulated. With OCT guidance, canaloplasty was performed successfully by nonglaucoma specialists. During surgery, the collector vessel was located with real-time OCT imaging, achieved with the MIOCT system. Moreover, a scleral flap was precisely created above the collector vessel. With these collector vessels visible, a vertical incision was made at an appropriate depth. Unlike the routine approach, a deep scleral flap was not required (29). This makes the procedure much easier to perform and reduces potential complications.

Previously, canal expansion was only verified postoperatively by ultrasound biomicroscopy $(4,9)$. In this study, the placement of the microcatheter and the expansion of the collector vessel were confirmed in real time with MIOCT, which adds to the confidence of the surgeon while injecting the viscoelastic.

Formerly, Siebelmann et al. and Pasricha used intraoperative OCT for the visualization or monitoring of anti-glaucoma surgery, and both found it to be helpful and valuable. However, in their studies and other reports, it was noticed that the manner in which OCT images were projected needed improvement $(20,30)$ and it was suggested that the SS-OCT with a deeper penetration and high imaging speed might have great potential $(20,30,31)$. Based on these findings, our system used SS-OCT with a 1,310 $\mathrm{nm}$ light source, and the high-resolution images acquired by the OCT and the microscope were projected on the screen side by side. The results indicated that the MIOCT system was helpful in monitoring and guiding canaloplasty. In addition, the problem of the scleral tissue shadow noticed in the former report using an $840 \mathrm{~nm}$ OCT device (20) did not affect the surgeons in our study.

Although the collector vessels in the porcine eye differ from Schlemm's canal in the human eye (32), all of the steps involved in canaloplasty were simulated in this study. Therefore, our results provided evidence that the novel MIOCT system might have a potential value for canaloplasty. The possible correlation between the intraoperative findings (the degree of canal expansion) and the postoperative prognosis (the attenuation of IOP) will be investigated in the future.

With this system, combined 3D microscope and OCT images were viewed on the same screen. Although this permitted high-resolution real-time OCT image guidance, the $3 \mathrm{D}$ system was complex and beyond the typical imaging routine of most surgeons. Therefore, surgeons will require additional training with their use. Furthermore, although the beacon light clearly indicates the OCT imaging site, when the eye moves during surgical manipulation, its position must be readjusted to the region of interest, as previously noted by Hahn et al. (6,33). Therefore, an automatic tracking system that focuses the OCT scan beam on the preferred target would be extremely valuable and should be developed in the future.

Using an animal model of canaloplasty, our study demonstrated that the MIOCT system could acquire realtime, intraoperative high-resolution images of the anterior segment, and the acquired images could be helpful to surgeons. Future clinical trials will continue to demonstrate how the MIOCT system can be used in real practice.

\section{Acknowledgments}

The language of the manuscript was checked and modified by a professional medical writing service company (Editage, https://www.editage.cn/).

Funding: This article was supported, in part, by research grants from the National Key Research \& Development Plan (2017YFC0108200, 2017YFC0108201); the Shanghai Committee of Science and Technology (19441900900); Jiangsu Province Key Research \& Development Program (BE2018667).

\section{Footnote}

Reporting Checklist: The authors have completed the ARRIVE reporting checklist. Available at http://dx.doi. org/10.21037/atm-20-3469

Data Sharing Statement: Available at http://dx.doi. org/10.21037/atm-20-3469

Conflicts of Interest: All authors have completed the ICMJE uniform disclosure form (available at http://dx.doi. 
org/10.21037/atm-20-3469). The authors have no conflicts of interest to declare.

Etbical Statement: The authors are accountable for all aspects of the work in ensuring that questions related to the accuracy or integrity of any part of the work are appropriately investigated and resolved. The study was approved by the Institutional Review Board of the Eye and ENT Hospital of Fudan University, Shanghai, China (No. 2019031-1). Experiments were performed in accordance with the Association for Research in Vision and Ophthalmology Statement for the Use of Animals in Ophthalmic and Vision Research.

Open Access Statement: This is an Open Access article distributed in accordance with the Creative Commons Attribution-NonCommercial-NoDerivs 4.0 International License (CC BY-NC-ND 4.0), which permits the noncommercial replication and distribution of the article with the strict proviso that no changes or edits are made and the original work is properly cited (including links to both the formal publication through the relevant DOI and the license). See: https://creativecommons.org/licenses/by-nc-nd/4.0/.

\section{References}

1. Kingman S. Glaucoma is second leading cause of blindness globally. Bull World Health Organ 2004;82:887-8.

2. Weinreb RN, Aung T, Medeiros FA. The Pathophysiology and Treatment of Glaucoma: A Review. JAMA 2014;311:1901.

3. Francis BA, Singh K, Lin SC, et al. Novel Glaucoma Procedures. Ophthalmology 2011;118:1466-80.

4. Lewis RA, von Wolff K, Tetz M, et al. Canaloplasty: circumferential viscodilation and tensioning of Schlemm's canal using a flexible microcatheter for the treatment of open-angle glaucoma in adults: interim clinical study analysis. J Cataract Refract Surg 2007;33:1217-26.

5. Xin C, Tian N, Li M, et al. Mechanism of the reconstruction of aqueous outflow drainage. Sci China Life Sci 2018;61:534-40.

6. Lewis RA, Von Wolff K, Tetz M, et al. Canaloplasty: Three-year results of circumferential viscodilation and tensioning of Schlemm canal using a microcatheter to treat open-angle glaucoma. J Cataract Refract Surg 2011;37:682-90.

7. Khaimi MA, Dvorak JD, Ding K. An Analysis of 3-Year Outcomes Following Canaloplasty for the Treatment of
Open-Angle Glaucoma. J Ophthalmol 2017;2017:1-7.

8. Bull H, von Wolff K, Körber N, et al. Three-year canaloplasty outcomes for the treatment of open-angle glaucoma: European study results. Graefes Arch Clin Exp Ophthalmol 2011;249:1537-45.

9. Brusini P. Canaloplasty in open-angle glaucoma surgery: a four-year follow-up. ScientificWorldJournal 2014;2014:469609.

10. Brusini P, Caramello G, Benedetti S, et al. Canaloplasty in Open-angle Glaucoma: Mid-term Results From a Multicenter Study. J Glaucoma 2016;25:403-7.

11. Yalvac IS, Sahin M, Eksioglu U, et al. Primary viscocanalostomy versus trabeculectomy for primary open-angle glaucoma: three-year prospective randomized clinical trial. J Cataract Refract Surg 2004;30:2050-7.

12. Riva I, Brusini P, Oddone F, et al. Canaloplasty in the Treatment of Open-Angle Glaucoma: A Review of Patient Selection and Outcomes. Adv Ther 2019;36:31-43.

13. Dayani PN, Maldonado R, Farsiu S, et al. Intraoperative use of handheld spectral domain optical coherence tomography imaging in macular surgery. Retina 2009;29:1457-68.

14. Ehlers JP, Kernstine K, Farsiu S, et al. Analysis of pars plana vitrectomy for optic pit-related maculopathy with intraoperative optical coherence tomography: a possible connection with the vitreous cavity. Arch Ophthalmol 2011;129:1483-6.

15. Chavala SH, Farsiu S, Maldonado R, et al. Insights into Advanced Retinopathy of Prematurity Using Handheld Spectral Domain Optical Coherence Tomography Imaging. Ophthalmology 2009;116:2448-56.

16. Binder S, Falkner-Radler CI, Hauger C, et al. Feasibility of intrasurgical spectral-domain optical coherence tomography. Retina 2011;31:1332-6.

17. Ray R, Barañano DE, Fortun JA, et al. Intraoperative Microscope-Mounted Spectral Domain Optical Coherence Tomography for Evaluation of Retinal Anatomy during Macular Surgery. Ophthalmology 2011;118:2212-7.

18. Ehlers JP, Tao YK, Farsiu S, et al. Visualization of realtime intraoperative maneuvers with a microscope-mounted spectral domain optical coherence tomography system. Retina 2013;33:232-6.

19. Heindl LM, Siebelmann S, Dietlein T, et al. Future prospects: assessment of intraoperative optical coherence tomography in ab interno glaucoma surgery. Curr Eye Res 2015;40:1288-91.

20. Siebelmann S, Cursiefen C, Lappas A, et al. Intraoperative Optical Coherence Tomography Enables Noncontact 
Imaging During Canaloplasty. J Glaucoma 2016;25:236-8.

21. Kumar RS, Jariwala MU, V SA, et al. A Pilot Study on Feasibility and Effectiveness of Intraoperative SpectralDomain Optical Coherence Tomography in Glaucoma Procedures. Transl Vis Sci Technol 2015;4:2.

22. Li X, Wei L, Dong X, et al. Microscope-integrated optical coherence tomography for image-aided positioning of glaucoma surgery. J Biomed Opt 2015;20:76001.

23. Carrasco-Zevallos OM, Viehland C, Keller B, et al. Review of intraoperative optical coherence tomography: technology and applications [Invited]. Biomed Opt Express 2017;8:1607.

24. Miyazaki M, Shibuya K, Tokue H, et al. Percutaneous transhepatic biliary drainage assisted by real-time virtual sonography: a retrospective study. BMC Gastroenterol 2013;13:127.

25. Grüntzig AR, Senning Å, Siegenthaler WE. Nonoperative Dilatation of Coronary-Artery Stenosis: Percutaneous Transluminal Coronary Angioplasty. N Engl J Med 1979;301:61-8.

26. Tzeng BC, Wang CJ, Huang SW, et al. Doppler Ultrasound-guided Percutaneous Nephrolithotomy: A Prospective Randomized Study. Urology 2011;78:535-9.

27. Neoptolemos JP, London NJ, James D, et al. Controlled trial of urgent endoscopic retrograde

Cite this article as: Xu H, Fang W, Liu G, Fan J, Yu J, Zong Y, Jiang C, Shi G, Sun X. Feasibility of microscope-integrated swept-source optical coherence tomography in canaloplasty. Ann Transl Med 2020;8(23):1577. doi: 10.21037/atm-20-3469 cholangiopancreatography and endoscopic sphincterotomy versus conservative treatment for acute pancreatitis due to gallstones. Lancet 1988;2:979-83.

28. Davids AM, Pahlitzsch M, Boeker A, et al. Ab interno canaloplasty (ABiC)-12-month results of a new minimally invasive glaucoma surgery (MIGS). Graefes Arch Clin Exp Ophthalmol 2019;257:1947-53.

29. Cagini C, Peruzzi C, Fiore T, et al. Canaloplasty: Current Value in the Management of Glaucoma. J Ophthalmol 2016;2016:7080475.

30. Ang BCH, Lim SY, Dorairaj S. Intra-operative optical coherence tomography in glaucoma surgery-a systematic review. Eye (Lond) 2020;34:168-77.

31. Pasricha ND, Bhullar PK, Shieh C, et al. Fourdimensional microscope-integrated optical coherence tomography to enhance visualization in glaucoma surgeries. Indian J Ophthalmol 2017;65:57-9;

32. McMenamin PG, Steptoe RJ. Normal anatomy of the aqueous humour outflow system in the domestic pig eye. J Anat 1991;178:65-77.

33. Hahn P, Carrasco-Zevallos O, Cunefare D, et al. Intrasurgical Human Retinal Imaging with Manual Instrument Tracking Using a Microscope-Integrated Spectral-Domain Optical Coherence Tomography Device. Transl Vis Sci Technol 2015;4:1. 


\section{Supplementary}

Table S1 The intraclass correlation coefficient (ICC) values for the measurement of perimeter, area and long diameter of collector vessel before and after canaloplasty between the two readers

\begin{tabular}{|c|c|c|c|c|c|c|}
\hline & \multicolumn{2}{|c|}{ Perimeter } & \multicolumn{2}{|c|}{ Area } & \multicolumn{2}{|c|}{ Long diameter } \\
\hline & Before & After & Before & After & Before & After \\
\hline ICC & $0.992^{*}$ & $0.888^{*}$ & $0.988^{\star}$ & $0.993^{\star}$ & $0.996^{\star}$ & $0.984^{\star}$ \\
\hline
\end{tabular}

*Statistical values were calculated from the reliability analysis $\left({ }^{\star} \mathrm{P}\right.$ $<0.05)$

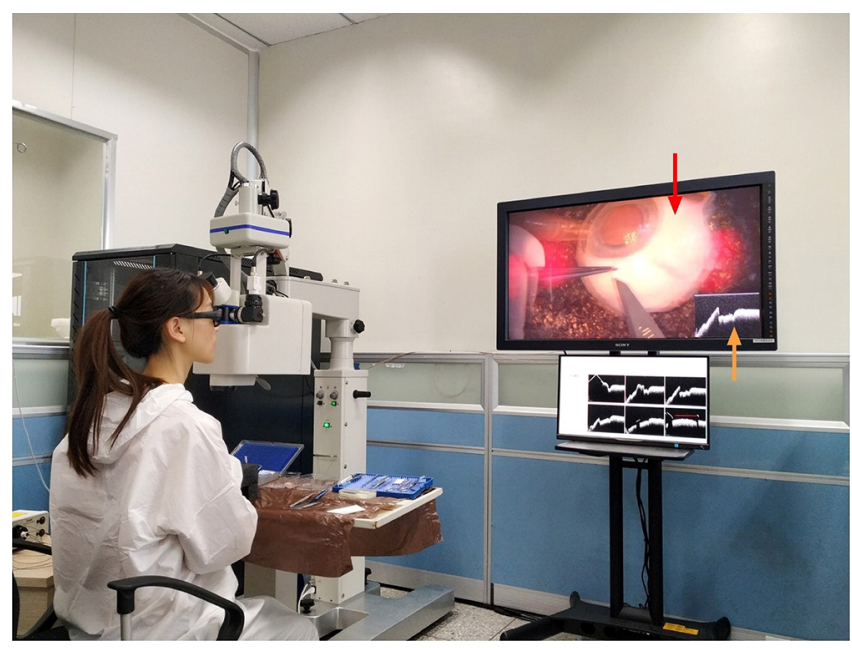

Figure S1 The microscope integrated optical coherence tomography (MIOCT) and external 3D head-up visualization system. The MIOCT images (yellow arrow) and surgical microscopic images (red arrow) are simultaneously projected on the external 3D head up display.

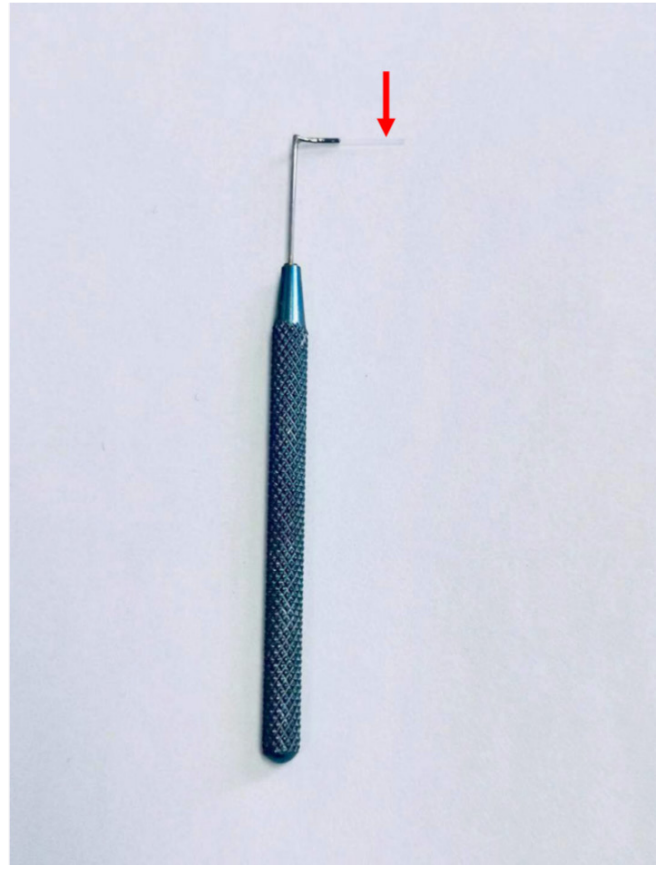

Figure S2 A tailor-made trabeculotome with a tip (red arrow) adapted from light fiber $(\Phi=250 \mu \mathrm{m})$. 